\title{
Floating Drug Delivery System: An Editorial Note
}

\author{
Someshwar Komati ${ }^{1}$, Suryakanta Swain ${ }^{\star 2}$, Muddana Eswara Bhanoji Rao ${ }^{3}$ and Vishali Das ${ }^{1}$ \\ ${ }^{\prime}$ Department of Pharmaceutics, University College of Pharmaceutical Sciences, Palamuru University, Mahaboobnagar, Telangana, India \\ ${ }^{2}$ Southern Institute of Medical Sciences, College of Pharmacy, Mangaldas Nagar, Vijyawada Road, Guntur-522 001, Andhra Pradesh, India \\ ${ }^{3}$ Department of Pharmaceutics, Roland Institute of Pharmaceutical Sciences, Khodasinghi, Berhampur-760 010, Ganjam, Odisha, India
}

\section{Article Info}

\author{
*Corresponding author: \\ Suryakanta Swain \\ Southern Institute of Medical Sciences \\ College of Pharmacy, Mangaldas Nagar \\ Vijyawada Road, Guntur-522 001, Andhra \\ Pradesh, India \\ Tel.: +91-9438038643 \\ Fax: +91-680-2404112 \\ Email: swain_suryakant@yahoo.co.in
}

Received: June 10, 2017

Accepted: June 27, 2017

Published: July 5, 2017

Citation: Komati S, Swain S, Bhanoji Rao ME, Das V. Floating Drug Delivery System: An Editorial Note. Madridge J Nov Drug Res. 2017; 1(1): 10-11.

doi: 10.18689/mjndr-1000102

Copyright: (c) 2017 The Author(s). This work is licensed under a Creative Commons Attribution 4.0 International License, which permits unrestricted use, distribution, and reproduction in any medium, provided the original work is properly cited.

Published by Madridge Publishers
Keywords: Floating, Drug delivery, Absorption and Bioavailability.

\section{Importance of open access and special features of the journal}

Open access plays a pivotal role in propagating scientific information globally. It is completely user friendly and is open for all to access easily. Its salient features are, that it covers 50 worlds's leading languages and have the facility of audio versions too. It invites every individual to share as well as explore scientific knowledge and research in the field of pharmaceutical sciences. It enhances scientific skills of the authors, readers, reviewers, editors and advisors by providing scientific credits. On whole it encourages each and everyone and attracts all towards the majestic world of science.

\section{Structure of the editorial}

Medicine is not only a science; it is also an art. It does not consist of compounding pills and plasters; it deals with the very processes of life, which must be understood before they may be guided. Many fascinating discoveries have been made in the field of pharmacy to help mankind get rid of day-to-day problems as well as fast spreading diseases. Oral control release delivery systems are the ones which deliver the drug at predetermined rate, locally or systemically, for a specified period of time. It is the most widely used delivery systems among all. But many adversities such as short gastric residence times and unpredictable gastric emptying times, differences in gastric physiology have affected the drug delivery behaviour. This lacuna made the scientists formulate novel delivery systems which retained in the stomach for prolonged and predictable period of time. there are many Approaches to achieve gastric retention such as,low density system or Floating delivery that cause buoyancy in gastric fluid, Mucoadhesive system that causes bioadhesion to stomach mucosa, Swell able system or extendible, which limits emptying, High density system or sinking system that is retained in the bottom of the stomach, Super porous hydro gel system, Magnetic system. Floating drug delivery systems (FDDS) solved all problems cited. Floating drug delivery system (FDDS) is also called as Hydro dynamically balanced system (HBS). It has a bulk density which is less than gastric contents and hence remains buoyant in the stomach for a prolonged period of time without affecting the gastric residence time. It has many advantages such as The bioavailability is significantly enhanced, Targeted therapy for local ailments in the upper G.I.T, reduction of fluctuation in drug concentration, Minimizing systemic exposure of drugs, eliminate side effects, Enables reduced frequency of dosing with improved patient compliances.

Floating drug delivery system (FDDS) have a bulk density less than gastric fluids and so remain buoyant in the stomach without affecting the gastric emptying rate for a 
prolonged period of time. While the system is floating on the gastric contents, the drug is released slowly at the desired rate from the system. After release of drug, the residual system is emptied from the stomach. This results in an increased GRT and better control of fluctuation in plasma drug concentration. However, besides a minimal gastric content needed to allow the proper achievement of the buoyancy retention principle, a minimal level of floating force $(\mathrm{F})$ is also required to keep the dosage form reliably buoyant on the surface of the meal. To measure the floating force kinetics, a novel apparatus for determination of resultant weight has been reported in the literature. The apparatus operates by measuring continuously the force equivalent to $F$ (as a function of time) that is required to maintain the submerged object. The object floats better if $\mathrm{F}$ is on the higher positive side. This apparatus helps in optimizing FDDS with respect to stability and durability of floating forces produced in order to prevent the drawbacks of unforeseeable intragastric buoyancy capability variations.

There are some factors that should be taken into consideration which has significant effect on the gastric retention time. Firstly physiological factors such as density of dosage form, it should be less than the gastric contents about $1.004 \mathrm{gm} / \mathrm{ml}$, shape of the dosage form with diameter more than $7.5 \mathrm{~mm}$ are reported to have increased GRT, size of the dosage form should be Tetrahedron and ring shaped devices with a flexural modulus of 48 and 22.5 kilo pounds per square inch (KSI) are reported to have better GRT $90 \%$ to $100 \%$ retention at 24 hours compared with other shapes. Then comes biological factors such as Fed or Unfed State, Nature of Meal, Caloric content, Frequency of feed, Age, Posture, Gender and disease state of the patient. A dosage form when designed needs to be safe, efficacious and stable. The following are the approaches to design a FFDS that is single-unit dosage form and multiple-unit dosage form. Based on the mechanism of buoyancy, two distinctly different technologies have been utilized in development of FDDS.

\section{Application of floating drug delivery system}

Floating drug delivery offers several applications for drugs having poor bioavailability because of the narrow absorption window in the upper part of the gastrointestinal tract. It retains the dosage form at the site of absorption and thus enhances the bioavailability. For Sustained drug delivery HBS system can remain in the stomach for long periods and hence can release the drug over a prolonged period of time. The problem of short gastric residence time encountered with an oral controlled release formulation, hence, can be overcome with these systems. These systems have bulk density of $<1$, as a result of which they can float on the gastric contents. For Site specific drug delivery These systems are particularly advantageous for drugs that are specifically absorbed from stomach or the proximal part of the small intestine e.g. Riboflavin furosemide and misoprostal and lastly for absorption enhancement drugs that have poor bioavailability because of site specific absorption from the upper part of the GIT are potential candidates to be formulated as floating drug delivery systems, there by maximizing their absorption.

I would like to thanks the Editor-in-chief of journal for the invitation, for giving me opportunity for writing an invited 2 pages editorial article for publication in the upcoming issue. 